Case We present the case of a 41-year-old MSM who was admitted with fever, confusion and agitation following unprotected anal sex 6 weeks earlier with a man of unknown HIV status. He required intubation due to the level of agitation. Admission CT and MRI brain were unremarkable. The first test HIV test was weakly antibody positive; a repeat 6 days later showed a stronger antibody response consistent with HIV seroconversion. His baseline CD4 count was 138 (18\%); HIV viral load was 929000 copies/ml. CSF analysis showed 4 white blood cells, elevated protein at $1.2 \mathrm{~g} / \mathrm{dl}$, and a normal CSF to plasma glucose ratio. There was insufficient CSF sample for HIV viral load testing. A diagnosis of encephalitis secondary to HIV seroconversion was made and antiretroviral therapy (ART) with five drugs was started on day 3 of admission. The patient remained agitated for several days. By day 21 the seroconversion symptoms had fully resolved, and by day 28 the plasma HIV viral load was undetectable. Viral genotyping showed the $\mathrm{K} 103 \mathrm{~N}$ mutation only. The patient remains on ART; now simplified to Kivexa, Darunavir and Ritonavir. This is to continue for 48 weeks at which point a decision will be made to either stop treatment or to continue lifelong ART.

Conclusion Transmitted drug resistant HIV can cause severe seroconversion illness and high levels of viraemia despite lower viral fitness. To our knowledge, this is the first report of viral encephalitis at HIV seroconversion caused by drug resistant HIV. The role of entry inhibitors and integrase inhibitors for the treatment of severe seroconversion symptoms to prevent viral entry into cells and aid rapid decline in viraemia are currently under evaluation.

\section{P173 CASE REPORT: RARE CAUSE OF ADULT ONSET SEIZURES IDENTIFIED IN AN HIV POSITIVE ADULT}

doi:10.1136/sextrans-2012-050601c.173

${ }^{1} E$ Pease, ${ }^{*}{ }^{1} S$ Dawson, ${ }^{2} \mathrm{G}$ Quaghebeur, ${ }^{1} \mathrm{~J}$ Ashby. ${ }^{1}$ The Garden Clinic; ${ }^{2} J o h n$ Radcliffe Hospital, Oxford, UK

Case Report A 63-year-old male with a long history of poor social interactions presented acutely confused. There was no alcohol or recreational drug use. Baseline bloods and routine microbiology were normal. CT and MRI of the brain found no causative pathology. Lumbar puncture (LP) only revealed a raised protein (1.47). An HIV test was positive; CD4 170 cells/ $\mu$ l, viral load (VL) 238281 copies/ $\mathrm{ml}$. The patient refused antiretroviral (ARV) treatment, depressive episode was diagnosed and mirtazapine commenced. Three days later he went into status epilepticus. A repeat MRI brain was unchanged and LP showed: protein 0.84; glucose 4; WBC 5/cmm; India ink, CRAG, virology, acid fast bacilli and cytology were negative. Seizures were unremitting and nevirapine and truvada were commenced and mirtazapine withdrawn: seizure activity ceased. Gradually he improved and his CD4 rose to 280 cells/ $\mu$ l, HIV $\mathrm{VL}<50$. Despite treatment, the patient re-presented with seizures on several occasions. No trigger was identified, and reported ARV adherence good. Repeat investigations revealed no new abnormality. In the absence of an adequate explanation for seizures, neuroimaging was reviewed by a specialist neuroradiologist. Nodules within the frontal horns of the lateral ventricles were identified, indicating a diagnosis of subependymal nodular heterotopia ( $\mathrm{SNH}$ ). Discussion This is the first report of SNH in an HIV positive adult. $\mathrm{SNH}$ are congenital, occurring during foetal neuronal proliferation and have no known association with HIV. Patients usually present in their 2nd decade with seizures and exhibit cognitive difficulties. $\mathrm{SNH}$ presenting in the 7 th decade of life with status epilepticus is rare and we postulate that the cerebral atrophy associated with untreated HIV infection may have precipitated seizures in a predisposed individual. This case described demonstrates the importance including non-HIV related causes, when considering the aetiology of seizures in patients with HIV.

\section{P174 THE TANGLED MESH OF LYMPHOGRANULOMA VENEREUM}

doi:10.1136/sextrans-2012-050601c.174

I Okpaluba, ${ }^{*}$ R J C Dunham, A Evans. Leeds Teaching Hospitals NHS Trust, Leeds, UK

Introduction The HPA report an epidemic of lymphogranuloma venereum (LGV) among men who have sex with men in the UK since 2003. Untreated LGV may lead to chronic or irreversible complications with disabling anatomic defects. Reports suggest surgical intervention is rarely required.

Case 45-year-old man who has sex with men, diagnosed HIV positive in 1995, not on antiretroviral therapy due to patient reluctance, following intolerance to several previous regimens. He had spent the summer in Egypt and on return to the UK had a brief spell as an inpatient with campylobacter and norovirus diarrhoea. He presented to the GUM clinic reporting multiple high risk partners and was treated as a gonorrhoea, chlamydia and syphilis contact; STI screen negative. He re-presented a month later with severe proctitis; pain and blood per rectum and frequent small volume stool. Rectal chlamydia was positive and subsequently LGV serovars confirmed. He tested positive for hepatitis $C$, retrospective sampling of stored blood samples suggested that this had been recently acquired. Hepatitis C treatment was unsuccessful due to lack of virological response and he suffered acute psychosis likely secondary to pegylated interferon. Despite 3 weeks of doxycyline and negative chlamydia retesting he persisted with severe proctalgia and constipation and was referred to gastroenterology. Flexible sigmoidoscopy showed a single ulcer in the upper third of the rectum with the remainder of the colon looking normal. He received empirical retreatment of LGV, rectal predfoam and laxatives. Symptoms worsened and a defecating proctogram (video of proctogram available) identified marked anorectal intussusception. This was surgically managed with a mesh support inserted laparoscopically.

Conclusion Despite early recognition, treatment and apparent clearance of LGV infection complications can occur. This is the first report of complicating anorectal intussusception requiring surgical intervention with a mesh support.

\section{P175 THE GREAT PRETENDER STRIKES AGAIN}

doi:10.1136/sextrans-2012-050601c.175

${ }^{1}$ T Moby, ${ }^{*}$ A Mwirigi, ' J White. 'St Thomas' Hospital; ' 2 University Hospital Lewisham, London, UK

Background An epidemic of syphilis persists in UK men who have sex with men (MSM), often with unusual manifestations. We report a case of syphilis mimicking a lymphoproliferative disorder.

Case A 29-year-old previously well MSM was admitted with 3 weeks of generalised painless lymphadenopathy. He reported malaise, night sweats and joint pain while travelling recently in the Middle East. He was apyrexial and routine bloods were normal apart from mildly raised liver enzymes. Paul Bunnell and HIV antibody tests were negative. His GP screened for STIs but did not include syphilis serology despite the patient describing penile lesions. Clinicians felt that the presentation was highly suggestive of lymphoma. A CT scan showed multiple enlarged lymph nodes in the neck and small bowel mesentery. An open cervical lymph node biopsy was performed. Histopathology showed suppurating granuloma in a reactive lymph node with no evidence of lymphoma. Stains for HIV p24, acid-fast bacilli and fungi were negative. The suggested differential included lymphogranuloma venereum (LGV), cat scratch disease and melioidosis. At GU medicine review he reported sex with multiple partners in the preceding 6 months. He had a blotchy maculopapular rash on his penis and scrotum, though this was treated as "fungal" by junior staff. Molecular tests for 
chlamydial and gonococcal infections were negative, including throat swabs. Treponema pallidum (TP) antibodies were positive, $\mathrm{RPR}=1: 256$. He was recalled and started on doxycycline for both secondary syphilis and possible LGV. Two weeks later his lymphadenopathy had greatly reduced in size. His original lymph node biopsy was retrieved and TP immunostaining was performed, which revealed a profuse infiltrate of spirochetes.

Conclusion This case highlights the need for clinicians from all specialities to be alert for the many clinical manifestations of syphilis currently prevalent in MSM. Wider use of TP immunostaining in relevant specimens is warranted.

\section{P176 SCARS OF VENUS...AND AESCULAPIUS}

doi:10.1136/sextrans-2012-050601c.176

S Stockwell, ${ }^{*}$ J van der Walt, J A White. Guy's and St Thomas' NHS Trust, London, UK

Background The lymphogranuloma venereum (LGV) epidemic continues unabated in UK men who have sex with men (MSM). Whatever its presentation, prompt recognition of LGV can be challenging.

Aim We describe a case of LGV inguinal syndrome identified too late to prevent major surgery.

Case report A 46-year-old HIV-positive MSM presented to A\&E on a Saturday with a 3-day history of a painful left groin lump. He was stable on Atripla with CD4 count $400 \mathrm{cells} / \mathrm{ml}$. He had a tender left inguinofemoral mass and the surgical team took him to theatre for suspected incarcerated femoral hernia. At operation they found instead a large necrotic lymph node and excised it. The patient self discharged the next day. Two weeks later he presented to the GU clinic as a syphilis contact. He mentioned his recent surgery and review of the histology report from the excised node showed "appearances consistent with LGV or cat scratch disease...also a vigorous periadenitis and syphilis should be considered." Abdominal examination revealed a $25 \mathrm{~cm}$ recent surgical scar in the left iliac region. There was penile oedema with sclerosing lymphangitis. A small round subpreputial ulcer was found; it was non-tender and had a rolled edge. There was no perianal or anal lesion. Dark ground microscopy from the ulcer was negative. Rectal and urethral swabs showed no pus cells. The patient was treated with 21 days doxycycline for suspected LGV as well as to cover early syphilis. Chlamydia trachomatis: RNA was detected from the ulcer, first catch urine and rectal specimens. The ulcer tested negative for herpes simplex and TP DNA. All gonococcal tests were negative. LGV-specific DNA was detected from the ulcer but not the rectal swab. TP antibodies were positive with an RPR titre of 4 . TP immunostaining of the node biopsy was negative. All clinical signs had resolved by the end of the 3-week doxycycline course.

Conclusion/Learning points This case highlights that LGV may still evade detection in both $\mathrm{A} \& \mathrm{E}$ and surgical departments. Awareness of LGV remains poor outside of GUM.

\section{P177 INVESTIGATING THE COST-EFFECTIVENESS OF INTEGRATED PRENATAL HIV/SYPHILIS SCREENING TO PREVENT ADVERSE PREGNANCY OUTCOMES IN CHINA}

doi:10.1136/sextrans-2012-050601c.177

K Owusu-Edusei, ${ }^{*}$ G Tao, T L Gift, Y Tun, M L Kamb, X Wei, L M Wang, M Bulterys. Centers for Disease Control and Prevention

Background The need to scale-up integrated prenatal HIV/syphilis screening in China has increased in the last decade owing to recent increases in syphilis and HIV cases.
Objectives In this study, we assess the health and economic outcomes of integrated $\mathrm{HIV} /$ syphilis screening to prevent adverse pregnancy outcomes in China.

Methods A Markov cohort decision analysis model was used to examine the health and economic outcomes of pregnancy using data from published literature and local data. Adverse pregnancy outcomes examined included miscarriage, induced abortion, congenital syphilis, stillbirth, low birth weight, mother-to-child HIV transmission and neonatal death. We examined four screening strategies; No-Screening, HIV-Only, Syphilis-Only and HIV-andSyphilis. We estimated Disability-adjusted life years (DALYs) for all health outcomes. The life expectancy of the child and mother were the analytic horizon.

Results Preliminary results indicated that for a cohort of 10000 pregnant women $(0.07 \%$ prevalence for HIV and $1.5 \%$ for syphilis; $20 \%$ of HIV + had syphilis), the HIV-Only strategy prevented 1 case of mother-to-child HIV transmission and resulted in 2 induced abortions; the Syphilis-Only strategy prevented 3 stillbirths, 2 miscarriages, 15 cases of congenital syphilis, 6 cases of low birth weight and 1 neonatal death, but resulted in 35 induced abortions. The HIV-and-Syphilis strategy prevented the sum of the outcomes prevented by the HIV-Only and Syphilis-Only strategies. Estimated costs per DALYs prevented were: Syphilis-Only, \$38; HIV-and-Syphilis, \$122; HIV-Only, \$5806.

Conclusions Integrated prenatal HIV/syphilis screening is more cost-effective than HIV-Only screening. This is largely due to the relatively higher prevalence of syphilis and the substantially lower cost of treatment.

\section{Other}

\section{P178 IS PUNISHMENT AND CRIMINALISATION OF HIV TRANSMISSION JUSTIFIED?}

doi:10.1136/sextrans-2012-050601c.178

M D Phillips, ${ }^{*}$ S Clegg. Bolton Centre for Sexual Health, Bolton, UK

Aim To examine why it may be acceptable to punish HIV positive individuals for unintentionally transmitting HIV through consensual sex

Method Key issues for criminal punishment include deterrence, retribution, rehabilitation and incapacitation. This framework was applied to the justification of criminal prosecution for non-intentional transmission of HIV through consensual sexual intercourse.

Results

Retribution This includes pain inflicted or rights removed (such as freedom) in response to an action. Criminal law is retributive. However, in England and Wales, prosecution occurs for "reckless transmission" not reckless endangerment. It is legal to take a chance, but illegal if a certain outcome occurs.

Deterrence A threatened legal sanction may prevent crime through fear of retribution. Criminalising transmission of HIV to an unknowing partner may increase disclosure. This approach is flawed. (1) The charge of reckless transmission needs the HIV positive person to know they are probably or definitely infected: criminalisation may prevent testing. (2) Criminalisation is counterintuitive to placing responsibility for an individual's sexual health with themselves, not with someone else. (3) Criminalisation stigmatises the HIV positive community.

Rehabilitation Rehabilitation in prisons is not perfect, with $39.3 \%$ of those with convictions being re-convicted of crime in 2009. Any change in behaviour in the absence of specialised programmes may be due to deterrence.

Incapacitation It is difficult to practise safer sex in prison as there may be a higher risk of coercion, sex work and covert sex. Sexual healthcare in the prison setting is unlikely to be equal to community 